\title{
Variation in Volume Production Through Clonal Deployment: Results From a Simulation Model to Minimize Risk for Both a Currently Known and Unknown Future Pest
}

\author{
By A. D. YanchuK ${ }^{1), *}$, J. BishiR ${ }^{2)}$, J. H. Russell ${ }^{3)}$ and K. R. Polsson ${ }^{1)}$
}

(Received 29 $9^{\text {th }}$ July 2005)

\begin{abstract}
A simulation model was developed to examine optimum patterns of deploying selected clones in the hypothetical situations of both a currently known pest and an unknown future pest. We modelled the interactions between Sitka spruce (Picea sitchensis (Bong.) Carr.), an economically important forest tree in British Columbia and the northwestern U.S., and the spruce terminal weevil (Pissodes strobi (Peck)), a major pest in western spruces. The model is combined with the Province of British Columbia's Tree and Stand Simulator (TASS) model to drive individual tree growth and stand establishment and development.

Two clonal-sampling strategies are examined: a randomly drawn set of genotypes or clones, to depict the potential consequences of a new (e.g., exotic) or a previously unimportant natural pest attacking a 'random' set of genotypes, and a 'fixed' set of clones, emulating a 'commercial' or known set of clones for growth and resistance mechanisms. Simulations use a range of numbers of genotypes or clones (2, 6, 18 and 30), and three deployment patterns (a random mixture of ramets, single-clone blocks, and a mosaic of smaller clonal blocks), in one and five hectare (Ha) stands. Total merchantable timber volume on a per $\mathrm{Ha}$ basis at harvest age 80 is used to compare the various combinations and schemes.

With both random and fixed chosen sets of clones, the random planting pattern (i.e., random mixture of ramets from the clonal set) produced the most volume. Eighteen randomly chosen clones generally produced more volume, than 2, 6 and 30 clones, but differences among 6 , 18 and 30 clones were small in most cases, irrespective of planting pattern. For fixed clones, the use of more resistant clones with higher growth potential produced more volume; however, pure clonal blocks of the best clone were not better than a mixture of that clone and an inferior one. Reducing the effects of insect activity and attack on trees, by lowering the average annual temperature in the model, or turning off all insect 'activity', increased merchantable volume but did not change the optimum number of clones $(\sim 18)$ or deployment pattern (random mixture). Forestry agencies can weigh these findings against economic advantages of block plantings of similar genotypes, in the choice of an appropriate number of clones and a deployment strategy.
\end{abstract}

1) Research Branch, British Columbia Ministry of Forests, $1^{\text {st }}$ Floor, 722 Johnson Street, Victoria B.C. V8W 1N1 Canada.

2) North Carolina State University, Department of Mathematics, Raleigh, North Carolina, USA 27695 - 8205.

$\left.{ }^{3}\right)$ Research Branch, British Columbia Ministry of Forests, Cowichan Lake Research Station, 7060 Forestry Road, Mesachie Lake, B.C. VOR 2N0.

*) Corresponding author: Phone: (250) 387-3338; Fax: (250) 3870046; E-mail: alvin.yanchuk@gov.bc.ca.
Key words: clonal forestry, genetic diversity, risk, merchantable volume loss, insect resistance.

\section{A. Introduction}

\section{A.1 Clonal forestry}

The development and deployment of genetically improved forest trees, either improved seedlings from traditional seed orchards or clones from clonal testing programs, has been under way for several decades. However, there are still many questions related to the 'best' approach to deploy them across the landscape. Best, in this sense, includes a number of factors such as potential 'practical' growth and yield issues (e.g., diameter class distributions, economic returns), and pest or disease losses to both current and future threats.

Germany and Sweden in particular have had regulations governing minimum numbers of clones that can be used (MuHs, 1993). These regulations are aimed primarily at protecting individual stands or larger regions from the dangers inherent in using only a few clones. They require minimum numbers of clones ranging from 20 to 500 , depending on the size of the planting programs in the region and the degree to which the clonal mixture has been tested (MuHs, 1993). LIBBY (1982), and others (BISHIR and RoBERDs, 1997, 1999; RoBERDs et al., 1990; ROBERDS and BISHIR, 1997) dissent, however, showing that the use of a large number of clones rarely provides a level of risk significantly different from that obtained from 20 to 40 clones.

These studies have been important in understanding the levels of genetic diversity in production populations that are necessary to minimize risk of loss. Risk, in this previous work, focuses on the probability that the proportion of planted stems that die before harvest exceeds a predetermined bound, while varying the numbers of clones (assuming simple genetic systems of traits providing resistance or susceptibility). However, these risk parameters are generally quite arbitrary and do not reflect biological and economic considerations for most forest stand management objectives.

Clonal block plantings have the theoretical advantages of decreasing inter-genotypic effects, but also improving the operational ease in rearing stock, plantation establishment and tracking, logging and processing, or even restoration in the face of a specific clonal failure (LIBBY and RAUTER, 1984; LIBBY, 1990). However, these advantages have not been well quantified in terms of growth and yield, and damage due to pests or pathogens and, to the best of our knowledge, incremental economic gains for many of these assumptions or practices have not been published. 
In this paper we extend the published research in an attempt to further quantify risks against currently known or expected threats by using a population dynamics model for a well characterized forest tree host/pest system. The results we report here confirm and refine the general conclusions of LIBBY (1982), Bishir and Roberds (1997, 1999), Roberds et al. (1990) and ROBERDS and BISHIR (1997). Interestingly, LINDGREN and PRESCHER (2005), recently reported similar values (to those mentioned above) for the number of genotypes required in seed orchard populations for Scots and loblolly pine, based on several different assumptions and genetic management goals. We address these issues in the context of spruce terminal weevil (Pissodes strobi (Peck)) phytophage interaction on spruce (Picea spp.).

Several features make this system attractive to investigate these particular questions. First, a substantial amount has been reported about this host-pest system (e.g., Alfaro, 1996; Dixon and Houseweart, 1982, 1983; GARA et al., 1971; TOMLIN et al., 2000). Second, genetic resistance is present (KISS and YANCHUK, 1991; KING et al., 1997; KING et al., 2004) and seems to exhibit several types of phenotypic resistances (Alfaro, 1996; Alfaro et al., 1997; SAHOTA et al., 1994; TOMLIN et al., 1997; TomLIN et al., 2000). If a few typical plant pest resistance mechanisms (e.g., attraction to the host, physical barriers to attack and chemical toxicity) can be modelled as a first approximation of a hierarchical system of resistances, a greater understanding of additive and non-additive effects of resistances can be applied to deployment issues. This may also allow us to generalize these findings even further, such that it could apply, for example, to the number of seed orchard parents required rather than simply the number of clones. Third, the insect we are considering, spruce terminal weevil, causes stem damage but not tree mortality per $s e$. Trees that are successfully attacked do not die, so less resistant clones may still contribute to final stand volume if damage has not been too severe. In turn, this may make our results conservative with respect to pest or disease infestations that cause outright mortality.

\section{A.2 Pest and genetic resistance in the stand- The case for modelling}

For risk due to currently unknown threats (e.g., introduction of an exotic pest), current deployment schemes are largely insurance designs based on considerations that are not pest or pathogen specific, but follow what one hopes is a general genetic pest/plant resistance model. In contrast, presence of a known risk (e.g., spruce weevils in Sitka spruce, white pine blister rust (Cronartium ribicola) in various five-needle pine species (Pinus spp.), southern pine beetle (Dendroctonus spp.) in loblolly pine (Pinus taeda)), which may cause damage and loss of growth, or mortality, can allow for models that account for specific host/pest interactions.

The deployment of clones for reforestation requires certain management decisions for optimizing wood production and minimizing risk, in particular: 1) the number of clones to be used and 2) the pattern in which to deploy clones (assuming species and site selection are decided). Is there a single best combination of these fac- tors, or does the optimal deployment pattern depend on the number of clones used? While several field studies are under way in British Columbia, the time required (e.g., 25-80 years, depending on species and location), make a modelling approach the only feasible short-term strategy for answering these questions in forestry. Moreover, it would not be possible to assess risk potentials, if in fact no threat materializes during the life of a particular set of field trials.

\section{B. The Model}

The population dynamics model we have developed (BISHIR et al., in preparation) consists of two biologically based parts, one simulating daily tree/insect interactions, the other reporting yearly tree mortality and growth, along with total merchantable volume of timber, both for the stand and for each clone. In this structure, host/pest interactions cause damage, and downstream economic implications are derived from the associated tree growth model and volume predictions. Due to the complexity of the model, only a brief summary is provided here.

\section{B.1 Tree planting, growth and competition}

Each simulation begins by assigning individual 'stems' in a rectangular grid. This planting is produced by generating a list of clones, then placing ramets from these clones onto the grid according to a preset pattern, as described in Sections C.3 and C.4. Planting of the stand, and subsequent tree growth and mortality, are simulated by the British Columbia Ministry of Forests' Tree and Stand Simulator (TASS), the primary growth and yield prediction model used for producing managed stand yield projections in British Columbia (MITCHELL and CAMERON, 1985). The basic biological principles used in the development of TASS are presented in MitcheLL (1975), and the computational approaches and uses of TASS appear in Mitchell et al. (1989). Alfaro et al. (1996) also used TASS to estimate the influence of different levels of insect attack on final timber yields.

Simulation models assist in answering complex questions that cannot be addressed by empirical methods. For instance, BURKHART et al. (1987), developed a tree growth model similar to TASS, and LIH et al. (1995), Coulson et al. (1989) and STEPHEN and LIH (1985), describe computer models that integrate southern pine beetle/loblolly pine interactions. PowELl et al. (1996), present a model of this genre for the mountain pine beetle/lodgepole pine system. All these models focus on pest spread and damage during a single season resulting in very dynamic and representative results of what may be going on in the field.

\section{B.2 Weevil/tree interaction}

Because weevils rarely attack very young stands, planted trees are first advanced through a weevil-free juvenile period of eight years. At the beginning of year nine, one of two initial weevil distributions is imposed: uniform over the stand or concentrated in one corner (further described in C.7). Weevil population dynamics movement between trees, changes on each tree in numbers of adult males, ovipositing and non-ovipositing 
females, and juveniles - and the tree damage caused by these weevils, are then simulated daily until tree harvest (at 80 years). Annual growth and mortality reports are output in early spring by TASS.

The biological model and computer code follow the descriptions of weevil dynamics in DiXON and HouseWEART (1982, 1983), GARA et al. (1971), Siller (1968), Stevenson (1967), Sullivan (1960) and Tomlin and BoRDEN (1996). For spruce weevil, vital rates - level of feeding activity of adults, rate of oviposition, rapidity of weevil development from egg to adult, etc. - are governed largely by temperature (Sullivan, 1960; MCMullen, 1976; Overhulser and Gara, 1981; Alfaro et al., 1997). In the model, the level of maturity of a juvenile is measured by the number of degree-days accumulated since the eggs were laid, a total of 888 degreedays above $7.2{ }^{\circ} \mathrm{C}$ being required for complete development on Sitka spruce (MCMULLEN, 1976; OvERHULSER and GARA, 1981).

At harvest time, TASS estimated merchantable timber on a per hectare $(\mathrm{Ha})$ basis, for the stand and for each clone separately. All main effects in the simulation (Section $\mathrm{C}$ below) are compared in terms of average total merchantable volume per Ha. Analysis of variance (PROC GLM, in SAS, 1999-2001) was used to determine the significance of main effects and interactions, for volume under the scenarios presented in Section C. All effects were considered fixed except the random effect when clones were randomly chosen (see C.2 and C.3). Standard deviations and $95 \%$ confidence intervals computed from independent replications of each scenario, are also provided in some figures to graphically depict levels of significance among the main effects. For comparison, control runs with no pests present provide a measure of the percent reduction in potential timber output attributable to weevils, as well as indicating whether patterns of deployment yield similar results with and without the damaging agent present.

\section{The Simulations}

The main inputs into each simulation include 1) stand size, 2) number of clones, 3) attributes and method of choosing clones, 4) deployment or planting pattern, 5) average annual temperature, 6) average site index for the stand, and 7) initial weevil distribution.

\section{C.1 Stand size}

In the simulations, stands (StandSize) are either one or five $\mathrm{Ha}$ in area, planted at densities of approximately 1000 stems per Ha. Most of the simulation results presented will be from the one Ha runs, as the five Ha simulations produced similar results. Nevertheless, some five Ha stand results are presented in order to show the effects that larger 'sinks' (i.e., clonal blocks of particularly susceptible clones) might have on rates of spread, damage and overall volume loss (particularly if the weevils are initially bunched in one corner - see C.7 below).

\section{C.2 Number of clones}

The numbers of clones (NumCln) used in the simulations were $2,6,18$ and 30 , which represent a broad range of clone numbers that are currently considered operationally feasible in most forestry organizations. One-clone deployment results are inferred from the block plantings of two clones. We did not use more than 30 clones, because the previously cited work by LIBBY (1982) and others, sets what we believe to be a practical upper limit, based on qualitative genetic criteria.

\section{C.3 Attributes and method of choosing clones}

Each clone is described by a set of four model parameters that determine its degree of susceptibility or resistance to weevil attack and its growth rate. These are:

- RES - rate of resin flow into egg and feeding holes created by weevils (on a scale of $0-10$, with 10 indicating very high flow)

- TOX - toxicity of resin to weevils $(10=$ nearly $100 \%$ toxicity)

- ATR - attraction of crown volatile odours to weevils (10 = extremely attractive)

- GRW - a TASS parameter that governs tree 'vigour'; in essence, the rate at which TASS will grow individual stems (ramets) in absence of weevil attack.

Clone parameters were either fixed (from Table 1) or chosen at random (from normal distributions outlined below). The resistance parameter values for 2,6 or 18 fixed clones were taken from Table 1 by beginning with clone \#1 and continuing until all needed clones for the particular run were included. For the sake of brevity of results from the fixed clone analyses, we only show the results of the two and six clone scenarios.

These fixed parameter combinations were chosen to provide clear distinctions among clones with different levels in the three resistance mechanisms and to allow

Table 1. - Values of resistance factors RES (resin flow), TOX (toxicity) and ATR (attraction) employed in the fixed clone scenarios. The 2-clone runs used the first two of these 18 clones, and the 6-clone runs the first six. The TASS growth parameter, GRW, is positively correlated with RES at an $r=0.37$ in this set of clones while, as mentioned in the text, $r=0.5$ in the random set of clones.

$\begin{array}{crrrr}\text { Clone Number } & \text { GRW } & \underline{\text { RES }} & \underline{\text { TOX }} & \underline{\text { ATR }} \\ 1 & & & & \\ 2 & 0.8 & 2 & 2 & 8 \\ 3 & 1.2 & 8 & 8 & 2 \\ 4 & 1.2 & 8 & 2 & 8 \\ 5 & 0.8 & 2 & 8 & 2 \\ 6 & 1.2 & 8 & 2 & 2 \\ 7 & 1 & 5 & 5 & 5 \\ 8 & 0.8 & 2 & 2 & 2 \\ 9 & 0.8 & 2 & 8 & 8 \\ 10 & 1.2 & 8 & 8 & 8 \\ 11 & 1.1 & 2 & 2 & 8 \\ 12 & 0.9 & 8 & 8 & 2 \\ 13 & 0.9 & 8 & 2 & 8 \\ 14 & 1.1 & 2 & 8 & 2 \\ 15 & 0.9 & 8 & 2 & 2 \\ 16 & 1 & 5 & 5 & 5 \\ 17 & 1.1 & 2 & 2 & 2 \\ 18 & 1.1 & 2 & 8 & 8 \\ & 0.9 & 8 & 8 & 8\end{array}$


study of the consequences of planting specific clone mixtures. Input values for these clones reflect low and high resistances, but not immunity or complete susceptibility (e.g., a value of 2 rather than 0 , and a value of 8 rather than 10). Clones \#6 and \#15 were duplicated by using the same 'average' fixed parameters in order to examine stochastic differences between two similar genotypes in the model simulations. The 30 clone scenario was not modelled when using fixed clones as the additional permutations that could have been developed from the three mechanisms did not add to our understanding of the 'epistasis' (the non-linear interactions they were attempting to emulate among and within mechanisms or loci) that we were interested in examining.

Randomly generated parameter values were drawn from normal distributions, with a mean of 5.0 and a standard deviation of 1.5 for RES, TOX and ATR $( \pm 3$ standard deviations gives a range from $0.5-9.5), \mu=1.0$ and $\sigma=0.1$ for GRW. In the code, GRW is correlated with RES (correlation coefficient arbitrarily set at 0.5) to emulate a hypothetical positive genetic relation between growth rate and rate of resin flow. Although a genetic correlation between GRW and RES has not been explicitly documented in the literature, it is known that faster growing spruce genotypes are more resistant (KING et al., 1997), and more resistant spruce genotypes typically produce more resin canals (ALFARO et al., 1997) even after artificial wounding (e.g., TOMLIN et al., 1997).

Heritabilities of RES, TOX and ATR were effectively one, as no environmental variation was added around the genotypic values assigned to a clone for these three resistance traits. However, with most resistance parameter values ranging from 2 to 8 , combined with differences in the net effects of each resistance factor (discussed later), the 'realized' heritabilities of RES, TOX and ATR were less than one. The clonal-mean heritability of 0.5 for GRW - which is a low-to-average 'repeatability value' for growth rate in many forest trees, was achieved by adding a randomly generated site 'noise' to each ramet's GRW value.

\section{C.4 Deployment pattern}

Once clones were chosen, one of three types of planting patterns (PlantPat) was used:

- $\underline{\text { Random }} \underline{\text { Mix }}$ (RM) - at each planting spot in the stand a ramet is chosen randomly from the previously generated list of fixed clones denoted in Table 1, or from a randomly generated list of clones.

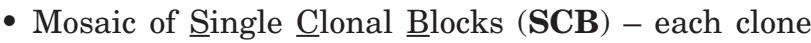
appears in only one block in the stand, each block containing the same number of stems.

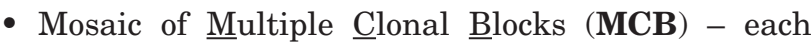
clone appears in two to six blocks (in any particular simulation, the number of blocks is the same for each clone), the blocks being of the same size and assigned to the various clones in a random order. In the one Ha runs of 18 and 30 random clones, MCB designs used only three blocks per clone, as otherwise it was not possible to create meaningful block sizes (i.e., for 18 clones there were $\sim 16$-tree blocks, and for 30 clones block sizes were very small at $\sim 9$-tree blocks). Therefore, the scenario of
MCB for one Ha with 30 random clones was not run in later analyses (i.e., data point is missing in Figures 6 and 7).

\section{C.5 Annual average temperature}

Temperature (Temp) is one of the main determinants of weevil feeding, oviposition and maturation rates. The rates used were determined from the work of GARA et al. (1971), McMullen (1976) and Overhulser and GarA (1981). Two annual average temperatures were used in the model $\left(7^{\circ} \mathrm{C}\right.$ and $11^{\circ} \mathrm{C}$; i.e., Temp $=7$ and 11) to examine how variations in weevil hazard activity influence stand outcomes. These levels were chosen to roughly reflect the diverse climates and growing conditions present in different areas of British Columbia (e.g., southern Vancouver Island compared to the north coast or coastal low and high elevation sites at the same latitude). In the simulations, daily averages within years followed a sine wave, around which simulated high and low temperatures were generated randomly, and each day's temperatures were positively correlated with the preceding day's average. Model equations incorporating temperature variation imposed effects on weevil dynamics, largely by slowing down their activity.

\section{C.6 Site index}

In our simulations, site index (SiteInd) indicates the expected average height of the stand, in meters, at age 60. Two site indices, SiteInd $=23$ and SiteInd $=30$, were used in the growth model, but for the reduction in SiteInd to 23 from 30, we also reduced Temp to 7 to better reflect the effect of temperature on both the trees and level of insect activity that would be more likely to occur in the field. These two SiteInd schemes were chosen to see if differences in growth rate affect the optimum pattern of deployment or the choice of number of clones.

\section{C.7 Initial weevil distribution in the stand}

At the beginning of year nine, one of two weevil 'introductions' or distributions of weevils (WeevDist) was imposed:

- Uniform, where each tree was 'assigned' three weevils at year nine (WeevDist $=1$ ), or

- Bunched, with 20 weevils per tree on 25 trees in one corner of the stand, with no weevils on any other trees (WeevDist $=2$ ).

The latter assignment simulates a more likely introduction pattern, by mimicking a small initial patch of weevils colonizing trees perhaps from a distant infested stand. A particularly relevant question is whether mixtures or blocks can act, on average, as traps or refugia for infestations, if these begin with a small number of weevils in a limited area of the plantation. WeevDist $=$ 0 denotes 'control' runs, in which no weevils were introduced into the stands.

\section{C.8 Variation in the simulations and outputs}

In total, we could have examined 2 (StandSize) $\times 4$ (NumCln) x 2 (fixed or random clones) x 3 (PlantPat) $x$ $2($ Temp $) \times 2($ SiteInd $) \times 2$ (WeevDist $)=384$ possible 
Table 2. - General linear model analysis of variance for StandSize (1 and $5 \mathrm{Ha}$ ), NumCln $=2,6,18$, and 30 , and PlantPat $($ RM, MCB, SCB $)$ when WeevDist $=1$, Temp $=11$, SiteInd $=$ 30 for randomly generated clones.

$\begin{array}{lrrrr}\text { Source } & \text { DF } & \text { Mean Square } & \text { F Value } & \text { Pr }>\text { F } \\ \text { StandSize } & & & & \\ \text { NumCln } & 1 & 17517.55 & 3.49 & 0.0630 \\ \text { PlantPat } & 3 & 34150.05 & 6.80 & 0.0002 \\ \text { StandSize*NumCln } & 2 & 209264.60 & 41.64 & <.0001 \\ \text { StandSize*PlantPat } & 3 & 20075.65 & 3.99 & 0.0083 \\ \text { NumCln*PlantPat } & 2 & 2918.93 & 0.58 & 0.5602 \\ \text { StandSize*NumCln*PlantPat } & 6 & 7462.93 & 1.48 & 0.1836 \\ \text { Error } & 6 & 4009.35 & 0.80 & 0.5724 \\ & 256 & 5025.58 & & \end{array}$

combinations of the seven stand attributes in Section C. However, since many of these are not feasible or are unlikely to be considered in actual forestry practices, we focused our simulations on a smaller subset of input combinations a manager can control, particularly the number of clones and the pattern in which they are deployed.

Variation between replications was achieved in two ways, depending on whether clones were chosen randomly or were fixed (i.e., picked from Table 1). In the latter case, clonal parameters remained the same in all replications of a particular scenario, and variation came from beginning each replication with a different seed for the randomly generated sequence of daily temperatures. Since variation is automatically present when clone parameters RES, TOX, ATR and GRW are generated randomly, we used the same seed for each temperature sequence, resulting in identical temperatures in all these replications. All scenarios were replicated 10-30 times and their average timber volumes compared. In total, approximately 1000 runs were completed with over 1000 hours of computing.

Timber volumes shown in Figures 1-7 depend not only on the parameters featured in this section, but also on values of other host-pest parameters programmed in the model (e.g., insect oviposition and maturation rates, mortality rates at each life stage, etc.). Thus, volumes presented in comparisons of deployment patterns, and in reported effects of temperature levels, weevil numbers, fixed clone parameter sets, etc., should be interpreted in a relative, not an absolute, sense. Note that the curved lines in Figures 1 to 7 are only used to provide the reader with an approximate interpolation between the data points for the random clones scenarios.

\section{Results}

\section{D.1 Randomly chosen clones}

Table 2 shows the analysis of variance results for the subset of inputs of Temp $=11$, SiteInd $=30$ and WeevDist $=1$ (these three parameter combinations will be referred to as the 'standard scenario' throughout the paper). Number of clones (NumCln), and planting pattern (PlantPat) were highly significant, while StandSize was marginally significant $(\operatorname{Pr}=0.06)$ (Table 2). Comparisons among the two stand sizes (StandSize $=1$ and $5 \mathrm{Ha}$ ) and the three planting patterns in Figure 1 show that the relatively low $\operatorname{Pr}>$ F value for StandSize in Table 2 is due primarily to differences in the $\mathbf{R M}$ deployment pattern versus the other two (i.e., SCB, MCB). Therefore, most of our results and discussion will be focused on StandSize $=1$, in order to reduce the number of comparisons presented. Although the StandSize x NumCln interaction was significant (Table 2), this also appears to be generated largely by NumCln = 2 , a number of clones unlikely to be used in practice (Figure 1). This was likely due to sampling errors that will occur with using only two clones and 10 replications in the simulations.

Among the NumCln values used, 18 clones produced the highest volumes at harvest (Figure 1). While the vol-

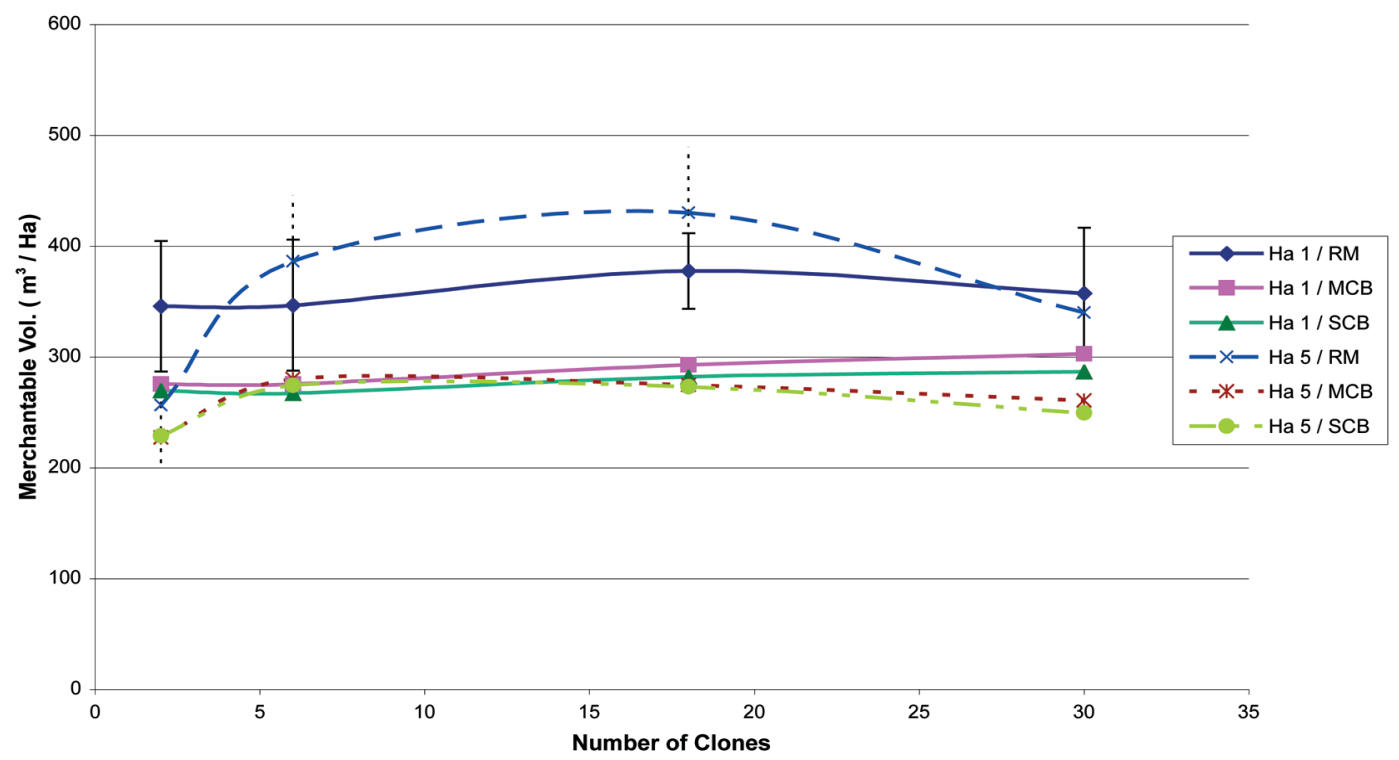

Figure 1. - Predicted merchantable volumes $\left(\mathrm{m}^{3} / \mathrm{Ha}\right)$ for combinations of StandSize (1 and $5 \mathrm{Ha}$ ), NumCln $(2,6,18$, and 30) for randomly selected clones, and PlantPat (RM, MCB, SCB). All simulations have WeevDist $=1$, Temp $=11$ and SiteInd $=30$. Error bars represent $95 \%$ confidence limits around the mean for RM scenarios only (5 Ha has dotted lines for error bars). 
ume differences for NumCln $=6$ and 18 are much higher in StandSize = $5 \mathrm{Ha}$ than for the StandSize = $1 \mathrm{Ha}$, the differences are not significant (Table 2). The RM scenario was consistently superior to the two clonal block planting patterns (i.e., MCB or SCB), averaging about $20 \%$ more merchantable volume (Figure 1). The MCB scenarios were usually better than the SCB, but these differences were small and not significant.

Although NumCln was significant, and the 18-clone scenario typically had higher yields in most of the comparisons in Figure 1, the differences among 6, 18 and 30 clones were not large within their respective PlantPat. Thus, even though there is considerable error around the means, and although 18 clones appear to be an 'optimum', there is a considerable range around this number that appears to give similar final volume. Once again, increasing the number of clones past 18 (e.g., 30 in our analysis), on average, does not offer greater protection against volume production loss.

In general, when individual clones were ranked by their cumulative stem volume, for StandSize $=1 \mathrm{Ha}$ the top $50 \%$ of the clones typically produced about $85 \%$ of the total volume of the stand (Figure 2). This skew in final contributing volume by the top clones causes a proportional reduction in the net representation of other clones, and therefore a reduction in genetic diversity over time. The loss in 'genotypic representation' can be evaluated by several measures of effective population size $\left(\mathbf{N}_{\mathbf{e}}\right)$ (HARTL and CLARK, 1989), one of which can be estimated by inverting the sum of the squared proportions of surviving ramets for each clone. This would essentially be the inbreeding effective population size; however, in our case it is also represents a measure of the loss of heterozygosity in the stand in the next generation (over its life, or if left to reproduce). This is partic- ularly relevant if we are interested in knowing if there would be a loss of genes that may be involved in the hypothetical resistance mechanisms we employed. For the NumCln = 30 scenario, the $\mathbf{N}_{\mathbf{e}}$ on average went to 24.8, NumCln $=18$ dropped to $\mathbf{N}_{\mathbf{e}}=14.7$, and NumCln $=6$ moved to $\mathbf{N}_{\mathbf{e}}=5.3$, even though one half of the clones produced most of the final crop in all three scenarios. These changes represent an approximate $20 \%$ reduction in effective population size from the time of establishment to the final crop, across all clone numbers used. With WeevDist $=0$, i.e., no weevils and normal intraand inter-genotypic competition at work, the reduction in $\mathbf{N}_{\mathbf{e}}$ was surprisingly only $\sim 3 \%$ higher for $\mathbf{N u m C l n}=$ 30 and 18, and almost the same for NumCln $=6$ and 2 (Figure 2).

To quantify the relationships among the growth and resistance parameters we programmed into the model, we used the Numcln $=30$ random clones and the 'standard scenario' (i.e., Temp $=11$, SiteInd $=30$, WeevDist = 1) run to determine standardised regression coefficients (i.e., path coefficients (LI, 1977)) between clone mean merchantable volume and the corresponding mean values for RES, TOX, ATR, and GRW. Path coefficients between merchantable volume and GRW, RES,

Table 3. - General linear model analysis of variance for StandSize $=1 \mathrm{Ha}$, NumCln $=2,6$ and 18 (from Table 1), PlantPat (RM, MCB, SCB $)$, WeevDist $=1$, Temp $=11$, and SiteInd $=$ 30 for fixed clones scenario.

$\begin{array}{lrrrr}\text { Source } & \text { DF } & \text { Mean Square } & \text { F Value } & \operatorname{Pr}>\text { F } \\ \text { NumCln } & & & & \\ \text { PlantPat } & 2 & 1426968.30 & 365.48 & <.0001 \\ \text { NumCln*PlantPat } & 2 & 2615768.43 & 669.96 & <.0001 \\ \text { Error } & 121 & 324218.47 & 83.04 & <.0001 \\ & & 3904.34 & & \end{array}$

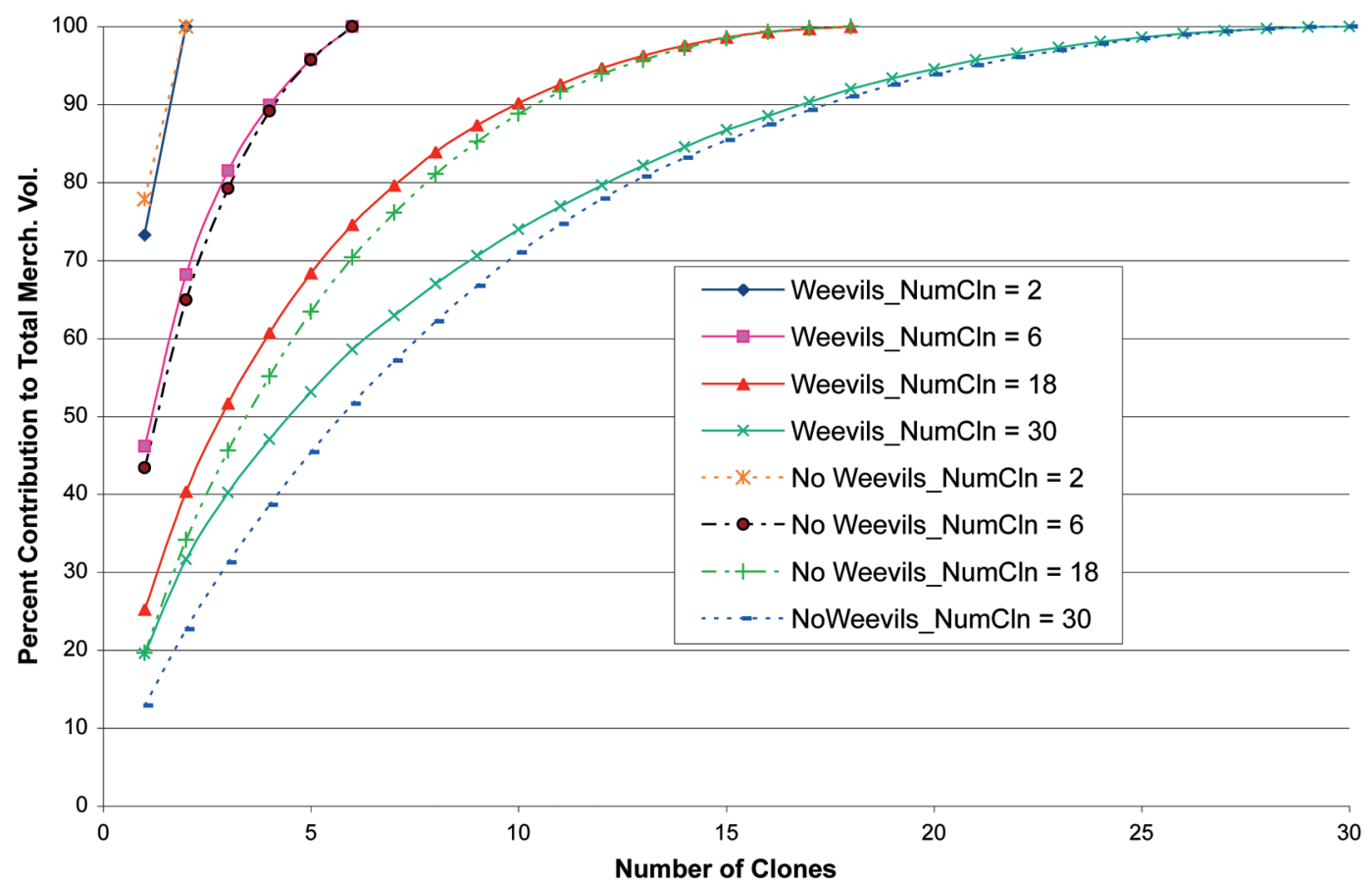

Figure 2. - Mean cumulative percent contribution to stand volume of randomly selected clones ranked from best to worst, for NumCln (2, 6, 18 and 30) for $1 \mathrm{Ha}$, WeevDist (0, NoWeevils and 1, Weevils), Temp $=11$, SiteInd $=30$. For each ranking, the value is the average of 30 independent runs. 


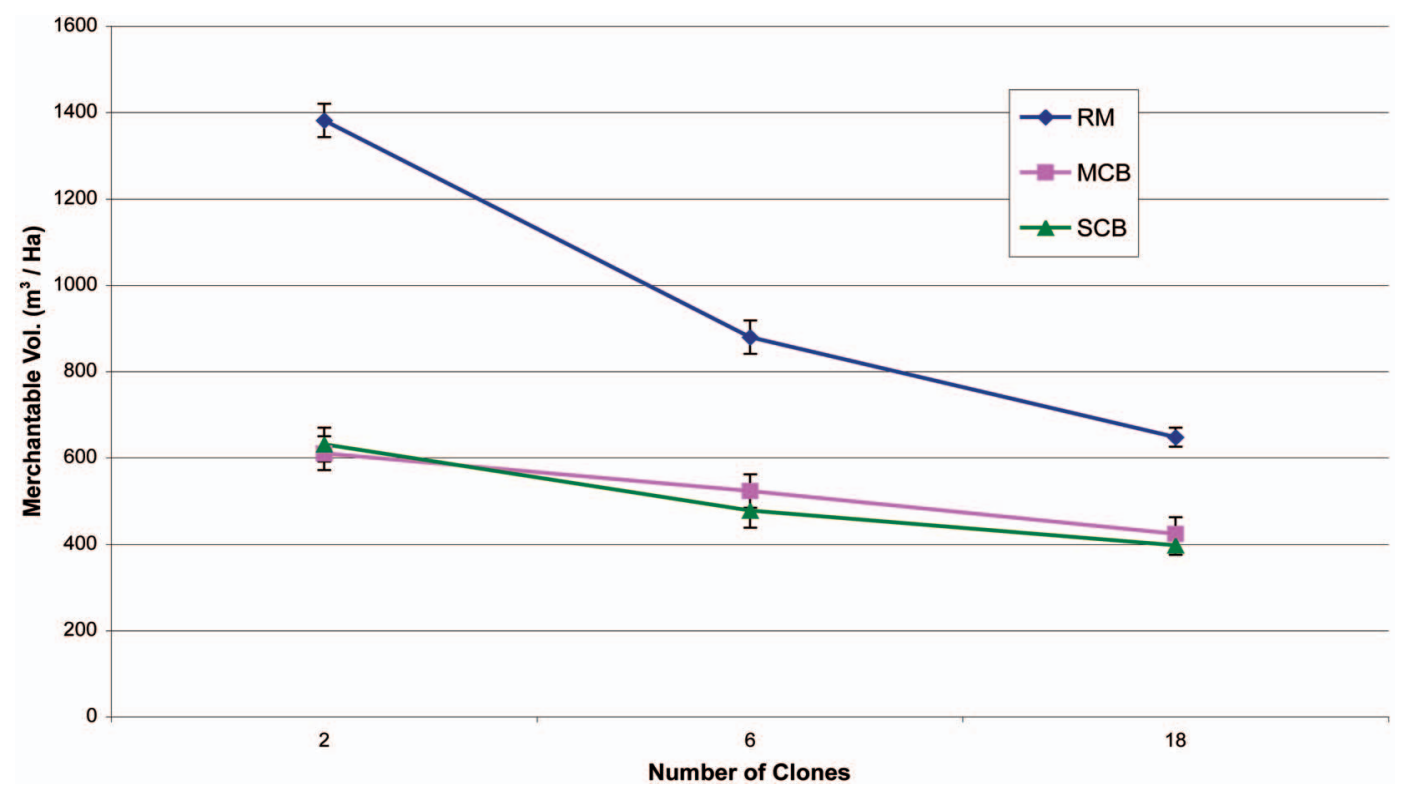

Figure 3. - Predicted merchantable volumes $\left(\mathrm{m}^{3} / \mathrm{Ha}\right)$ for StandSize $=1 \mathrm{Ha}$ and NumCln $(2,6$, and 18) fixed clones (Table 1) and for the three PlantPat (RM, MCB, SCB), WeevDist = 1, Temp = 11, and SiteInd $=30$ scenarios. Error bars represent $95 \%$ confidence limits around the mean.

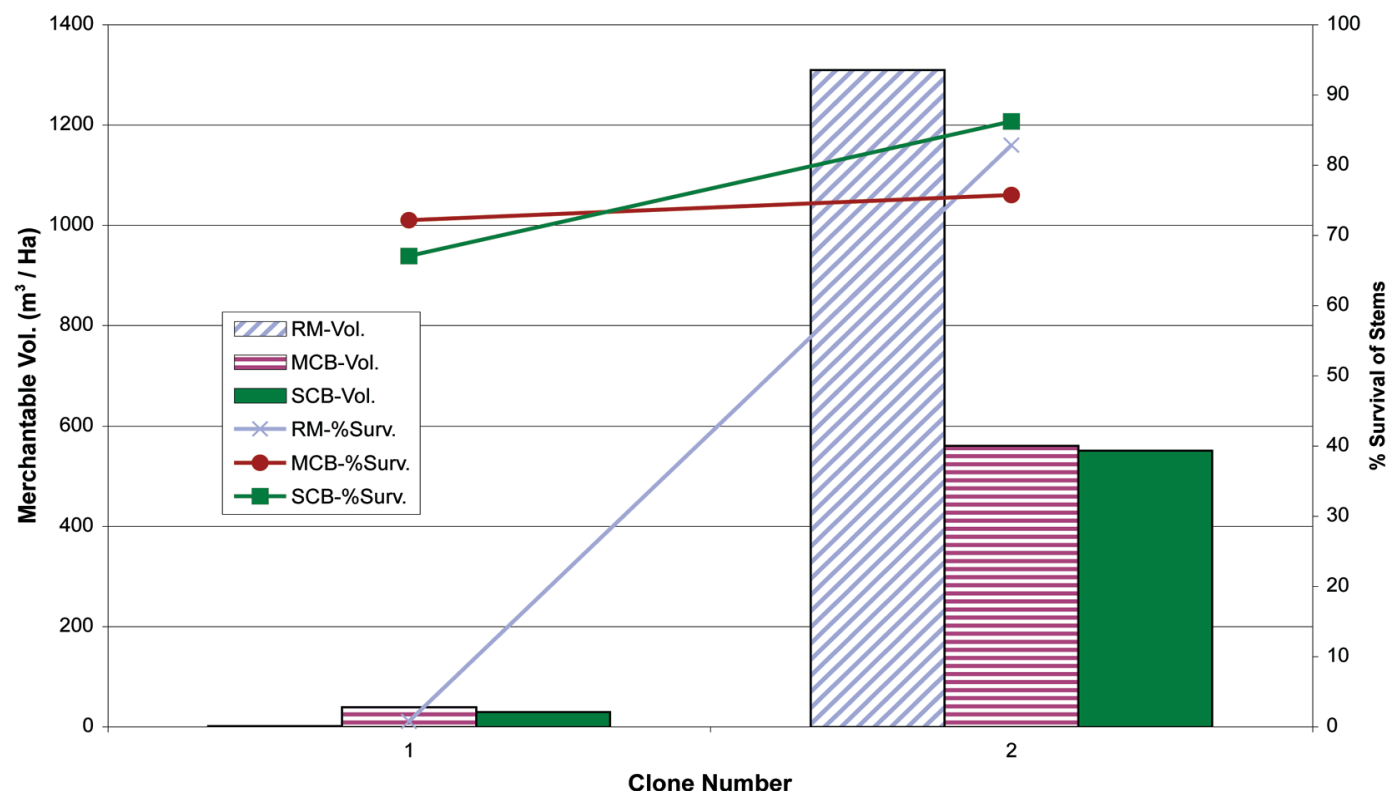

Figure 4. - Predicted merchantable volumes $\left(\mathrm{m}^{3} / \mathrm{Ha}\right)$ and percent survival of stems for StandSize = 1 Ha, fixed clones \#1 and \#2 (from Table 1), PlantPat (RM, MCB, SCB), WeevDist = 1, Temp = 11, and SiteInd $=30$.

TOX and ATR, were 0.26, 0.10, 0.08 and -0.59 , respectively, which indicate that GRW and ATR had the largest impacts on individual clonal performance in this model.

\section{D.2 Clones with fixed resistance and growth attributes}

For the fixed clone analyses and the 'standard' scenario (i.e., $\mathbf{T e m p}=11$, SI $=30$ and WeevDist $=1$ ), volume differences were significant and $\sim 30-50 \%$ higher with the RM than for the SCB and MCB (Table 3, Figure 3). Although the NumCln = 2 scenario was superior to the 6- and 18-clone scenarios, this was due to the fact that clone \#2, the fastest growing and most resistant of the 18 clones, was paired with the most susceptible clone (\#1) (Figure 4). Due to the comparative inferiority of other clones relative to clone \#2, the only valid comparisons among fixed clone scenarios are those involving the same number of clones (i.e., comparisons among NumCln $=2,6$ and 18 are not valid as clone \#2 is not in the same proportions across the three scenarios). Therefore, we only graphically present the 2- and 6-clone scenario below as the relative performance of different clones was similar in the 18-clone scenarios.

\section{D.2.1 Two clones}

As indicated above, the deployment of the NumCln = 2 in the RM planting pattern resulted in the greatest volume, compared to the other two patterns (Figure 3), 
but this was due to the almost complete mortality of clone \#1 (less than 1\% survival; clone \#2 had 80\% survival after 80 years) (Figure 4 ). Although $\sim 70 \%$ of clone \#1 trees survived after 80 years in the two-clonal block planting scenarios (i.e., SCB and MCB), volume differences between the two clones were still large (Figure 4). We can also infer from Figure 4 that a pure clonal block of clone \#2 (the SCB for clone \#2) might be inferior to a mixture of clones \#1 and \#2. The volume of clone \#2 in the SCB in Figure 4 is actually the total per clone on 0.5 $\mathrm{Ha}$ (however, for $\mathbf{R M}$ and $\mathbf{M C B}$, the $\mathrm{y}$-axis is on a $\mathrm{m}^{3} / \mathrm{Ha}$ basis); so even if we doubled this volume, as a matter of comparison, it would still fall surprisingly short of the volume of clone \#2 in the RM (i.e., $1309 \mathrm{~m}^{3}$ in the $\mathbf{R M}$ versus $550.1 \times 2=1100 \mathrm{~m}^{3}$ in the $\left.\mathbf{S C B}\right)$.

\section{D.2.2 Six clones}

Although the RM produced greater volume (Figure 3), when broken down by individual clones we see that this was primarily due to the performance of the two most resistant clones, \#2 and \#5 (Figure 5). SCB's were slightly lower in volume than MCB's (Figure 3), but this varied depending upon which clone was examined (Figure 5). The NumCln = 6 scenario offers an opportunity to examine interactions among the resistance mechanisms in the model. For instance, only for clones \#2 and \#5 were there noticeable differences between the SCB and MCB (Figure 5). For the more susceptible clones, there was very little difference in the net result from either SCB or MCB. In terms of survival of stems in the RM, clone \#1 had 21\% survival, whereas clone \#3 had only $35 \%$ survival, even though it had a better growth rate and higher resin flow than clone \#1 (Table 1). Clone \#5 (high growth rate, high resin flow and low attraction, making it the second most resistant clone), had high survivorship, at $84 \%$, even though it had a low toxicity value. Likewise, clone \#4 produced almost twice as much volume as clone \#3 (albeit a small contribution to final stand volume) even though it had a slow growth rate and a low resin flow value, and only slightly better survival rate than clone \#3 (Figure 5). The 'average' clone (\#6), with all resistance parameters equalling 0.5 and a GRW value of 1.0 , had $31 \%$ of its ramets survive through to rotation, about 20 percent lower than clone \#4, which had a lower growth potential, but good resistance parameters (i.e., $\mathbf{T O X}=8$ and $\mathbf{A T R}=2$ ).

\section{D.2.3 Eighteen clones}

For NumCln $=18$, nine of the clones produced more volume in the RM planting pattern than in the SCB and there were essentially no differences in ranking among the top six clones for volume production in either the RM or SCB scenarios (not shown). The two 'duplicate' clones (\#6 and \#15) had almost identical yields, indicating that the model performed the resistance and growth parameter profiles of clones very well (not shown).

\section{D.3 No weevil attacks and randomly selected clones}

In order to examine if the same general results hold up with regards to optimum patterns of deployment and numbers of clones without a damaging agent, we also ran the standard simulation scenario with no weevils present (WeevDist $=0$ ). We present results for the random clone scenarios only.

Although differences between weevil and no-weevil runs in the standard scenario were large in terms of volume production, i.e., about four times the volume relative to WeevDist $=1$, the general results as they related to the question of deployment were very similar (i.e., comparing Figures 1 and 6). Only NumCln and Plant-

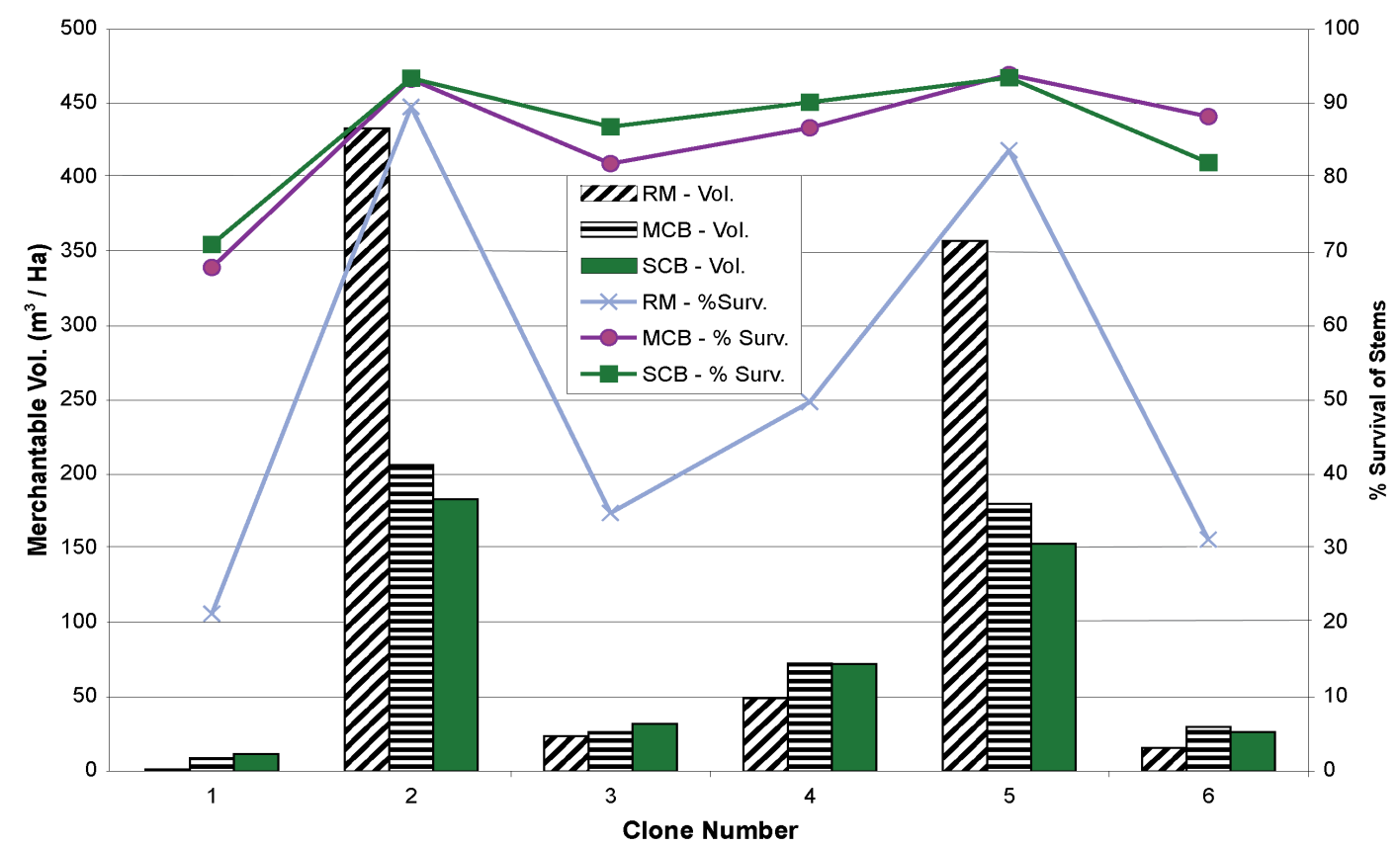

Figure 5. - Predicted merchantable volumes $\left(\mathrm{m}^{3} / \mathrm{Ha}\right)$ and percent survival of stems for StandSize of $1 \mathrm{Ha}$, fixed clones \#1 through \#6 (from Table 1), PlantPat (RM, MCB, SCB), WeevDist = 1, Temp = 11 , and SiteInd $=30$. 
Table 4. - General linear model analysis of variance for Standsize $=1$ and 5 Ha, NumCln $=2,6,18$, and 30, PlantPat (RM, MCB, SCB), WeevDist $=0$ (no weevils), Temp $=11$, SiteInd $=30$ for randomly generated clones.

$\begin{array}{lrrrr}\text { Source } & \text { DF } & \text { Mean Square } & \text { F Value } & \operatorname{Pr}>\text { F } \\ \text { StandSize } & & & & \\ \text { NumCln } & 1 & 347.41 & 0.03 & 0.8543 \\ \text { PlantPat } & 3 & 387569.63 & 37.64 & <.0001 \\ \text { Standsize*NumCln } & 2 & 451924.42 & 43.89 & <.0001 \\ \text { Standsize*PlantPat } & 3 & 8709.76 & 0.85 & 0.4693 \\ \text { NumCln*PlantPat } & 2 & 842.59 & 0.08 & 0.9214 \\ \text { Standsize*NumCln*PlantPat } & 4 & 6136.30 & 0.60 & 0.6657 \\ \text { Error } & 4 & 191.17 & 0.02 & 0.9993 \\ & 420 & 10296.15 & & \end{array}$

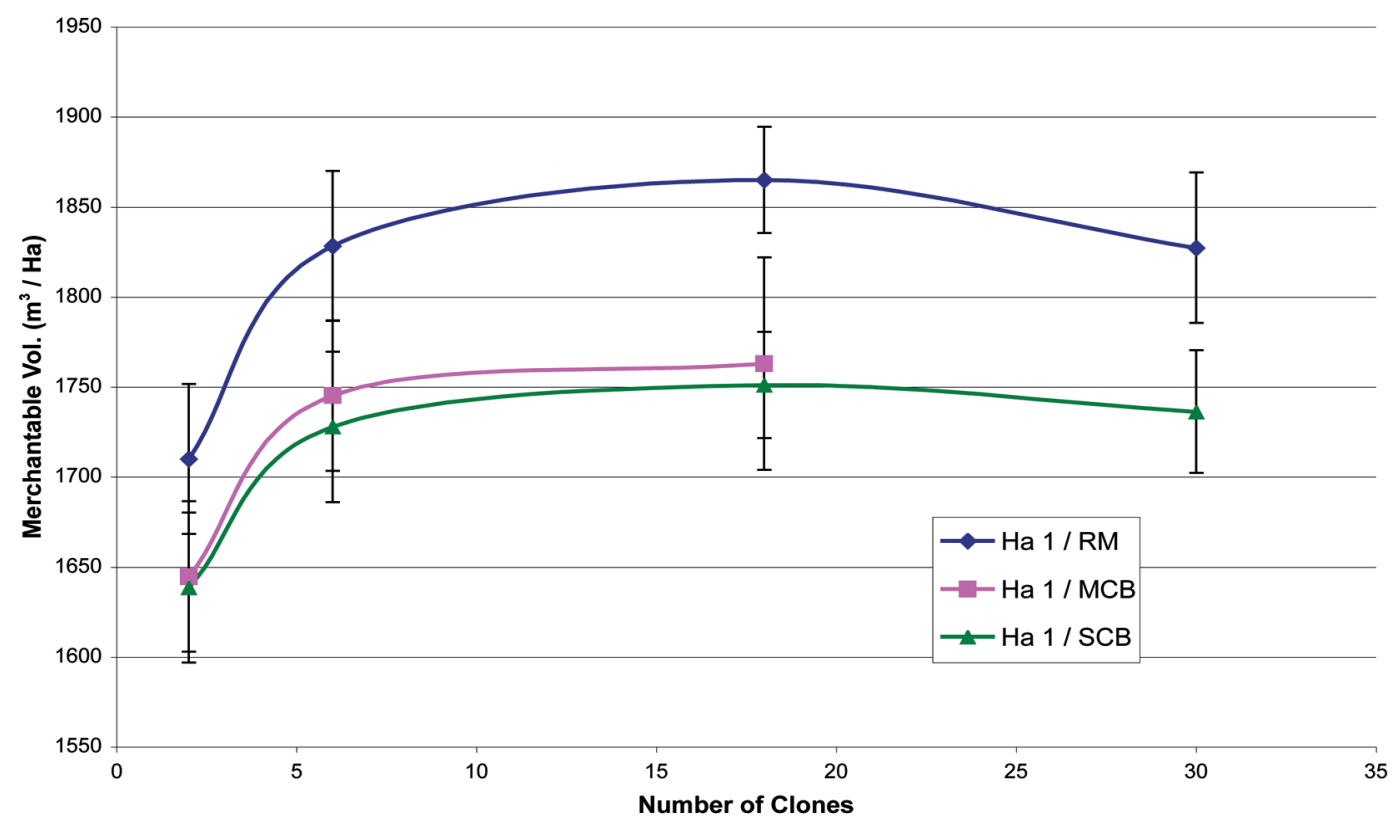

Figure 6. - Predicted merchantable volumes $\left(\mathrm{m}^{3} / \mathrm{Ha}\right)$ for NumCln $(2,618$ and 30$)$ randomly selected clones, PlantPat (RM, MCB, SCB), and WeevDist $=0$ (no weevils). All simulations have StandSize = $1 \mathrm{Ha}$, Temp $=11$, and SiteInd $=30$ scenario. Error bars represent $95 \%$ confidence limits around the mean.

Pat were statistically significant (Table 4). With no weevils present NumCln = 18 was again best for all planting patterns (Figure 6), but the $95 \%$ confidence interval bars again have a large overlap with NumCln $=6$ and 30 results. NumCln = 2 was inferior to the other three NumCln options we examined, and again, there was a slight decrease in merchantable volume for NumCln = 30 relative to $\mathbf{N u m C l n}=18$.

\section{D.4 Average annual temperature and site index}

As indicated earlier, we also examined the effects of different environmental conditions on weevil activity (as determined by different temperatures in the model), by lowering the average annual temperature from $11^{\circ} \mathrm{C}$ to $7{ }^{\circ} \mathrm{C}(\mathbf{T e m p}=7)$, and concomitantly lowering temperature to reflect a drop in site index (SiteInd) (i.e., we reduced SI to 23, from 30, resulting in slower tree growth).

Surprisingly, even though growth potential of the stand was lowered by a reduced SI to 23 , there was, on average, a greater timber volume at Temp $=7$ than at Temp = 11 (compare Figure 1 versus Figure 7). In simu- lations having no weevils, on the other hand, average volume was about $35 \%$ less at Temp $=7$ than when Temp = 11 (compare Figure 6 versus Figure 7 ). In all scenarios, however, the best pattern in which to deploy clonal materials was still the $\mathbf{R M}$, and the best number of clones was again around 18 (Figure 7).

\section{D.5 Initial weevil distribution for randomly selected clones}

Not surprisingly, the much larger initial insect populations present in the uniform infestation (Weevdist $=$ 1) substantially decreased timber output, by about $55 \%$ of that for WeevDist $=2$ (data not shown). However, similar to the other results we obtained, RM was the best deployment pattern, and plantings of 18 clones generally resulted in the greatest volume, though differences among the four NumCln sets we used were typically small.

\section{E. Discussion}

Of the seven attributes discussed in Section C, only StandSize, NumCln and PlantPat lie under complete 


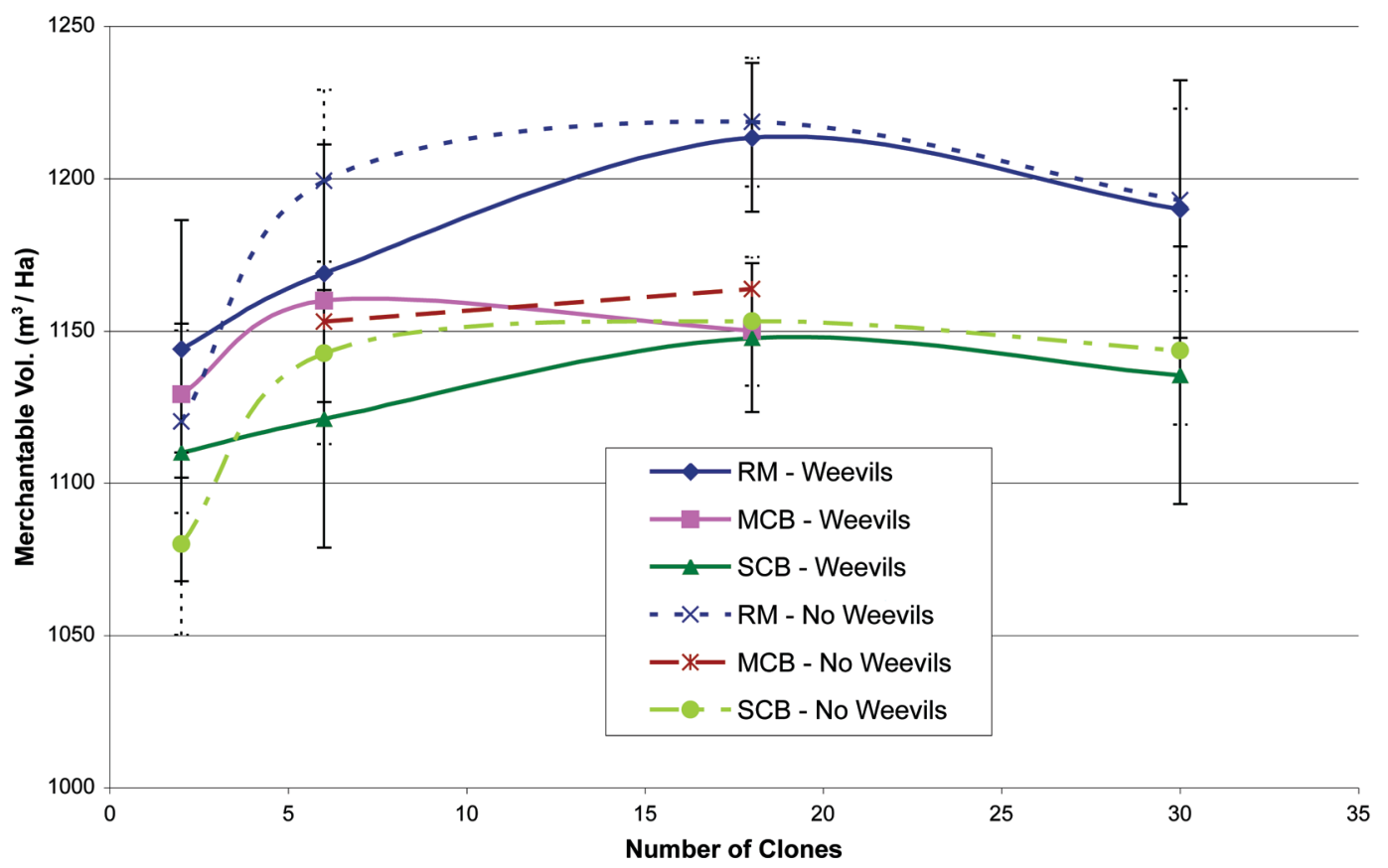

Figure 7. - Predicted merchantable volumes $\left(\mathrm{m}^{3} / \mathrm{Ha}\right)$ for StandSize $=1 \mathrm{Ha}$, for NumCln $(2,6,18$ and 30) randomly selected clones, PlantPat (RM, MCB, SCB), and WeevDist (0, No Weevils and 1 , Weevils). All simulations have Temp $=07$, and SiteInd $=23$ scenario. Dotted error bars represent 95\% confidence limits around the mean for 'No Weevils' scenarios, and solid error bars for the SCB only.

control of stand managers. Other attributes are largely under the influence of natural conditions of the forest environment. The results of this study suggest that a random mix of clones as opposed to clonal blocks, probably through competitive effects, allows better growing or more resistant genotypes to capture more physical space and ultimately increase their contribution to standing volume. How reflective this is for all forestry situations is of course unknown, but it does suggest that we need comparable field performance data to corroborate or refute our findings.

Almost independent of the number of clones chosen, whether they are fixed genotypes chosen for some currently known threat, or a random sample chosen to 'protect' against unknown threats (e.g., insect resistance as discussed in this paper), the greater the mixing of genotypes during plantation establishment, the better the overall response of the stand. In reality, however, for a set of fixed clones, a forest manager would prefer to select only clones like \#2 and \#5 and plant them exclusively, or at least would increase their proportions, to maximize volume. While this would be a logical decision, it is not clear that more volume would always be produced by keeping them separate in either the SCB or the MCB's, over the RM planting pattern. Furthermore, if the deployment strategy had to consider risk of a future pest (i.e., we then have to invoke the results of the random clone selection scenarios) we would have to conclude $\mathbf{R M}$ is again superior.

On average, approximately $85 \%$ of the volume came from the top $50 \%$ of the better growing and/or resistant genotypes deployed in a RM planting pattern. At the same time, there was usually a modest reduction in genetic diversity or effective population size $\left(\mathbf{N}_{\mathbf{e}}\right)$ over time in the stand. However, this 'inconsistency' between a relatively small reduction of genetic diversity $\left(\mathbf{N}_{\mathbf{e}}\right)$ and the proportion of stems that produce most of the final crop (i.e., the dominant and co-dominant stems that will have to endure any future biotic or abiotic challenges), suggests that policy and planning around genetic diversity in forest tree deployment should not entirely depend on measures or statistics like $\mathbf{N}_{\mathbf{e}}$ alone. On the other hand, it may be adequate simply to know there is an approximate reduction of $20 \%$ in final clonal representation in stands and plan accordingly. We fully expect that the effective number of mature individuals can and will change as a stand matures, but measures of genetic diversity should consider many facets of risk as well as the objectives of the forest manager. It appears from most of our analyses, that when the initial NumCln drops below six, there is more risk in terms of loss of potential volume. Interestingly, even with WeevDist $=0$ (i.e., no weevils present), the net clonal representations were very similar at rotation age with WeevDist $=1$ (i.e., weevils present) (Figure 2). This suggests that a damaging agent may play a relatively small part in creating an additional competitive effect in an otherwise normal stand of trees. In our simulations different clones have different 'fitness' characteristics due to growth and resistance traits, but the end result is that weevil damage only slightly reduced the overall genetic diversity in the stand.

The results also suggest that approximately 18 genotypes usually approach an 'optimum' for many conditions. Irrespective of a damaging agent being present or not, approximately 18 clones may achieve a balance between the number needed to sample enough good genotypes from some normal distribution of genotypic values, and have this random sample play out through stand development in their final net representation in a 
mature stand. Fewer clones than six, apparently do not sample enough of the genotypic distribution to assure inclusion of excellent clones, while more than 18 generally will include more superior genotypes but their net representation is smaller in the final stand. As mentioned earlier, the work of LIBBY (1982) and BISHIR and RoBERDs (1995, 1997, 1999), concludes that in general the level of risk is unlikely to change significantly after the number of clones exceeds 30 or 40 . Our results suggest a similar conclusion related to timber volume, based on a more complex set of risk parameters. Although we did not include more than 30 genotypes in our simulations, in most of the scenarios which we examined here, there was a noticeable drop in merchantable volume as we moved from use of 18 to 30 clones. It seems unlikely that volume production would increase at some point after 30 , and we conclude that no more than 30 clones are needed for either risk protection or near-optimal timber yield.

In terms of resistance mechanisms, the three putatively independent mechanisms we input into the model did generate some non-linear effects or interactions. Examination of the productivity of various fixed clones, together with path analyses coefficients, showed that ATR (attraction) and GRW (growth) were the two most important traits. Initially, we had no idea how the three resistance mechanisms would ultimately affect final 'resistance.' While the ATR resistance mechanism we chose may or may not be a biologically accurate reflection in this or any other host-pest system, it does allow us to evaluate the effects of stronger versus weaker mechanisms. In real-world tree-phytophage interactions, it is well known that there can be a few to many resistance mechanisms or factors present (KENNEDY and BARBour, 1992). Some mechanisms may exhibit a cost, and others, not (HERMs and MATTSON, 1992), but the net effects of all interacting mechanisms and growth potential will be complicated and difficult to predict (e.g., Pilson, 2000).

In our model we did not have any cost of resistance (i.e., a negative correlation between growth and any of the resistance mechanisms), but a benefit was coded into the model by the positive genetic correlation between RES and GRW which we speculated exists (AlfARo et al., 1997). Other permutations and combinations of genetic effects and correlations could be examined. However, it appears the net effect of any epistasis among mechanisms or growth will not affect the final result of our original question. We conclude this because, when the weevil model was 'turned off,' we had very similar results with respect to a range of the best number of clones and the deployment pattern. If a 'cost of resistance' is present (e.g., negative genetic correlations between growth and resistance traits), this could influence the above results and would have to be examined in separate studies. As we indicated earlier, the selection of fixed clone parameter values was intended to emulate a population of known clones identified through a clonal testing and selection program, in which: 1) many clones have high and similar growth potential, 2) all have some degree of resistance (i.e., none have resistance parameter values of 0 , and most have a high resistance score in at least one putative mechanism), and 3) a few susceptible and average clones are included to provide a 'realistic' situation that includes less desirable clones that reduce the chances of pest evolution. For each subset of clones tested in the simulation model, the RM always provided more merchantable volume than the two clonal-block scenarios we examined, regardless of stand size, initial weevil distribution or average annual temperature. However, the issue of whether a RM planting pattern or clonal blocks is better for reducing pest evolution is complex. Although recent studies in agriculture (e.g., DAVIS and ONSTAD, 2000; GOULD, 1986; STORER et al., 2003; TANG et $a l ., 2001)$ indicate the need for planting strategies that provide 'refugia' to safeguard against evolution of pests, it is highly dependent on the movement patterns of the insects.

If the four-degree $\mathrm{C}$ average temperature difference modelled in this study truly reflects an increase in insect hazard rating (i.e., Temp $=11$ and SiteInd $=30$ ), whether it be by naturally occurring 'hotspots', or by a general local increase through climate warming, it will have important implications, namely, an increase in the overall level of damage caused by insect activity. However, even with reduced temperatures and the concordant reduction in site index, deploying clones as random mixes still seems to be the best management decision. In the 'standard' scenario, merchantable volumes were approximately $1 / 4$ of the volumes present when WeevDist $=0$. While this may or may not be realistic with respect to what occurs in the field, we were simply attempting to cover some extreme cases to see if the results were consistent with respect to our forest management questions.

Although higher ratios of more resistant stock (e.g., using more of clone \#2) is appealing and could be an improvement, at some point (which would require more sensitivity analyses), there could be a reduction from some optimum combinations. Our results suggest that the use of a single best clone in a stand should be downplayed as, in even a pure block situation (i.e., SCB of clone \#2 in Figure 4), the RM allows more of the best clone(s) to attain dominant and co-dominant status on a per Ha basis. It appears that in the SCB's (or MCB's) intra-genotypic competition will be more prevalent, and at least from the model results we present here, causes significant losses to overall stand performance. Again, this seems to be a very robust result, as even when the weevil activity was not incorporated, the same results hold up with respect to NumCln $=18$ and with the RM planting pattern being the best.

The overall effects of the initial weevil distribution (WeevDist $=1$ or 2 ) also did not affect the results either with respect to optimum deployment patterns or the number of clones; however, with weevils introduced into the corner of the plantation, they obviously took time to spread through the plantation which had an overall positive effect on final timber output. With the RM planting pattern again being superior, it appears that, on average, clonal blocks, either as SCB or as MCB do not provide any additional protection or advantage with susceptible clonal blocks acting as 'sinks' and reducing insect 
spread. Although our WeevDist $=2$ scenario may be more realistic in the field (i.e., a few trees in the corner of the plantation are initially attacked), the general management implications are the same.

\section{F. Conclusions}

Our results suggest that mixtures of genotypes represent the best deployment strategy for clonally generated forest materials, as individual trees contribute to the stand development process and stems are lost through stand competition and mortality. As well, it appears that approximately 18 genotypes or clones approaches an 'optimum' or safe number for reducing risk to some unknown future biotic threat; however, the differences between 6 and 18 or 18 and 30 are relatively small. While our model, its parameter values, and the results may not be wholly applicable to all forestry situations, e.g., matching specific clones for specific site conditions, they may be general enough for many species where such detailed performance information is not available, or for those with longer rotation ages.

Clonal blocks have been attractive in forestry for many years, as they have the potential to provide uniformity in the growing, stock handling, and planting of selected clones (LIBBY and RAUTER, 1984; LIBBY, 1990). Such uniformity in large scale harvesting and processing of clonal blocks, as well as replacement options for a failed clone, may also be of economic value to the producer (LIBBY, 1990). Whatever the merits of selected clones in blocks in commercial plantations, inter-genotypic competition is important and we clearly need field experiments with long-term exposure to biotic threats, and including logging, handling, and processing, to assess economic advantages and to validate or invalidate our results. While our simulation approach and results answer some questions in clonal forestry, significant issues remain, particularly those related to landscape level deployment. However, it is reasonable to expect that if stand level diversity and planting patterns are optimum, then landscape level issues may largely need to focus on pest or disease evolution concerns (i.e., as discussed earlier, refugia to slow breakdown of resistance). Nevertheless, we believe these results provide some needed guidance for forestry agencies to weigh the economic advantages of block plantings versus random mixes, and the choice of an appropriate number of clones.

\section{Acknowledgements}

The authors would like to thank Michael STOEHR, JoHN KING and the two anonymous reviewers for the helpful comments on the manuscript.

\section{References}

Alfaro, R. I. (1996): Feeding and oviposition preferences of white pine weevil (Coleoptera: Curculionidae) on resistant and susceptible Sitka spruce clones in laboratory bioassays. Environ. Entomol. 25(5): 1012-1019.

Alfaro, R. I., R. G. Brown, K. J. Mitchell, K. R. Polsson and R. N. MACDONALD (1996): SWAT: A decision support system for spruce weevil management. In: Decision
Support Systems for Forest Pest Management - Proceedings of a workshop of the Entomological Societies of Canada and British Columbia, Oct. 17, 1995, Victoria, B.C. (Eds. Shore, T.L. and Maclean, D.A.). FRDA Report No. 260. pp 31-41.

Alfaro, R. I., F. He, E. Tomlin and G. Kiss (1997): White spruce resistance to white pine weevil related to bark resin canal density. Can. J. Bot. 75: 568-573.

BisHIR, J. and J. H. RoBERDS (1995): Analysis of time to failure in clonally propagated plant populations. Math. Biosci. 125: 109-125.

BISHIR, J. and J. H. RoBERDS (1997): Limit theorems and a general framework for risk analysis in clonal forestry. Math. Biosci. 142: 1-11.

BISHIR, J. and J. H. RoBERDS (1999): On numbers of clones needed for managing risks in clonal forestry. Forest Genet. 6: 149-155.

Bishir, J., K. R. Polsson, J. Russell and A. D. Yanchuk (2006): BC Weevil: A simulation model of the joint population dynamics between spruce weevil and Sitka spruce, over the lifetime of a plantation. (In preparation).

Burkhart, H. E., K. D. Farrar, R. L. Amateis and R. F. DANIELS (1987): Simulation of individual tree growth and development in loblolly pine plantations on cutover, site-prepared areas. Publication No. FWS-1-87, School of Forestry and Wildlife Resources, Virginia Polytechnic Institute and State University, Blacksburg, VA.

Coulson, R. N., R. M. Feldman, P. J. H. Sharpe, P. E. Pulley, T. L. Wagner and T. L. PAyne (1989): An overview of the TAMBEETLE model of Dendroctonus frontalis population dynamics. Holarctic Ecology 12: $445-450$.

DAVIS, P. M. and D. W. OnSTAD (2000): Seed mixtures as a resistant management strategy for European corn borers (Lepidoptera: Crambidae) infesting transgenic corn expressing Cry $1 \mathrm{Ab}$ protein. J. Econ. Entom. 93: 937-948.

Dixon, W. N. and M. W. Houseweart (1982): Life tables of the white pine weevil, Pissodes strobi (Peck), in central Maine. Environ. Entomol. 11: 555-564.

Dixon, W. N. and M. W. Houseweart (1983): Spring temporal and spatial activity patterns of adult white pine weevils (Coleoptera: Curculionidae) in Maine. Environ. Entomol. 12: 43-49.

GARA, R. I., R. L. CARLson and B. F. HRUtFioRd (1971): Influence of some physical and host factors on the behaviour of the Sitka spruce weevil, Pissodes sitchensis, in south-western Washington. Ann. Ent. Soc. Amer. 64: 467-471.

Gould, F. (1986): Simulation models for predicting durability of insect-resistant germplasm: Hessian fly (Diptera: Cecidomyiidae)-resistant winter wheat. Environ. Entomol. 15: 10-23.

Herms, D. A. and W. J. Mattson (1992): The dilemma of plants: to grow or defend. Quar. Rev. Biol. 67: 283-335.

HARTL, D. L. and A. G. Clark (1989): Principles of Population Genetics. Sinauer Assoc., Inc., Sunderland. 682 pp.

KenNEDY, G. G. and J. D. BARBOUR (1992): Resistance variation in natural and managed systems. In: Plant resistance to herbivores and pathogens. (Eds. FRITZ, R. S. and Simms, E. L.) pp. 13-43. Univ. Chicago Press, Chicago, Il.

KISS, G. and A. D. YANCHUK (1991): Preliminary evaluation of genetic variation of weevil resistance of interior spruce in British Columbia. Can. J. For. Res. 21: $230-234$. 
KInG, J. N., A. D. Yanchuk, G. KISS and R. I. Alfaro (1997): Genetic and phenotypic relationships between weevil resistance and height growth in spruce populations of British Columbia. Can. J. For. Res. 27: 732-739.

King, J. N., R. I. Alfaro and C. Cartwright (2004): Genetic resistance of Sitka spruce (Picea sitchensis) populations to the white pine weevil (Pissodes strobi (Peck)): distribution of resistance. Forestry 77: 269-278.

LI, C. C. (1977): Path analysis - a primer. $2^{\text {nd }}$ Ed. Boxwood Press, Pacific Grove. 347 pp.

LIBBy, W. J. (1982): What is a safe number of clones? In: Resistance to Disease and Pests in Forest Trees. (Eds. Heybroek, H., Stephan, B. and von WeissenberG, K.). Proceedings of the $3^{\text {rd }}$ International Workshop on the Genetics of Host-Parasite Interactions in Forestry. pp. 342-360. Wageningen, The Netherlands, Sept. 1980.

LIBBY, W. J. and R. M. RAUTER (1984): Advantages of clonal forestry. Forestry Chronicle. June, pp. 145-149.

LIBBY, W. J. (1990): Advantages of clonal forestry revisited. In: Section 5: Genetic Improvement, Vegetative Propagation: Proceedings of the Joint Meeting of Western Forest Genetics and IUFRO Working Parties S.2.02-05, -06, -12, -14, Douglas-fir, Contorta Pine, Sitka Spruce and Abies Genetic Research, 20-24 Aug. 1990, Olympia, WA. pp. 18-29.

LiH, M. P., F. M. StePhen and K. J. JeNG (1995): SPBMODEL A User's Guide. Offprint, Department of Entomology, University of Arkansas, Fayetteville, AR, USA.

Lindgren, D. and F. PREsCher (2005). Optimal clone numbers for seed orchards with tested clones. Silvae Genetica. 54: 80-92.

MCMullen, L. H. (1976): Effect of temperature on oviposition and brood development of Pissodes strobi (Coleoptera: Curculionidae). Can. Ent. 108: 1167-1172.

MitchelL, K. J. (1975): Dynamics and simulated yield of Douglas-fir. For. Sci. Monogr. 17. 39 pp.

MitChell, K. J. and I. R. CAMERON (1985): Managed stand yield tables for coastal Douglas-fir: initial density and precommercial thinning. Land Management Report No. 31. Province of British Columbia.

Mitchell, K. J., R. M. KellogG and K. R. Polsson (1989): Silvicultural treatments and end-product value. In: Second growth Douglas-fir: Its management and conversion for value. A report of the Douglas-fir Task Force, (Ed. KellogG, R. M.). Forintek Canada Corp. Spec. Publ. No. SP-32, Vancouver, B.C. pp. 130-167.

MuHs, H.-J. (1993): Policies, regulations and laws affecting clonal forestry. In: Clonal Forestry, Vol. 2 (Eds. AhuJA, M. and LiBby, W. J.) pp. 215-227. Springer-Verlag.

Overhulser, D. L. and R. I. Gara (1981): Site and host factors affecting the Sitka spruce weevil, Pissodes strobi (Peck), in western Washington. Environ. Entomol. 10: 611-614.

Pilson, D. (2000): The evolution of plant response to herbivory: simultaneously considering resistance and tolerance in Brassica rapa. Evol. Ecol. 14: 457-489.
Powell, J. A., J. A. LogAn and B. J. Bentz (1996): Local projections for a global model of mountain pine beetle attacks. J. Theor. Biol. 179: 243-260.

RoberDS, J. H., G. NAMKOONG and T. SkRoppa (1990): Genetic analysis of risk in clonal populations of forest trees. Theor. Appl. Genet. 79: 841-848.

RoBERDS, J. H. and J. BISHIR (1997): Risk analyses in clonal forestry. Can. J. For. Res. 27: 425-432.

SAHota, T., J. F. MANVILle and E. White (1994): Interaction between Sitka spruce weevil and its host, Picea sitchensis (Bong.) Carr.: A new mechanism for resistance. Can. Entom. 126: 1067-1074.

SAS Institute InC. (1999-2001): Release 8.02e. SAS Institute, Cary, North Carolina.

Silver, G. T. (1968): Studies on the Sitka spruce weevil, Pissodes sitchensis, in British Columbia. Can. Entom. 100: 93-110.

Stephen, F. M. and M. P. LIH (1985): A Dendroctonus frontalis infestation growth model: organization, refinement, and utilization, pp. 186-194. In: (Eds. BRANHAM, S. B. and Thatcher, R. C.). Integrated Pest Management Research Symposium: The Proceedings. U.S. Dept. of Agriculture Forest Service, Asheville, NC., USA, General Technical Report SO-56.

Stevenson, R. E. (1967): Notes on the biology of the Engelmann spruce weevil, Pissodes engelmannii (Coleoptera: Curculionidae), and its parasites and predators. Can. Ent. 99: 201-213.

Storer, N. P., S. L. Peck, F. Gould, J. W. VAN DuYn and G. G. Kennedy (2003): Spatial processes in the evolution of resistance in Helicoverpa zea (Lepidoptera: Noctuidae) to $\mathrm{Bt}$ transgenic corn and cotton in a mixed agroecosystem: a biology-rich stochastic simulation model. Jour. Econ. Ent. 96: 156-172.

Sullivan, C. R. (1960): The effect of physical factors on the activity and development of adults and larvae of the white pine weevil, Pissodes strobi (Peck). Can. Ent. 92: $732-745$

Tang, J. E., H. L. Collins, T. D. Metz, E. D. Earle, J. Z. ZhaO, R. T. Roush and A. M. Shelton (2001): Greenhouse tests on resistance management of $\mathrm{Bt}$ transgenic plants using refuge strategies. J. Econ. Entomol. 94: 240-247.

TOMLIN, E. S. and J. H. BoRDEN (1996): Feeding responses of the white pine weevil, Pissodes strobi (Peck) (Coleoptera: Curculionidae), in relation to host resistance in British Columbia. Can. Ent. 128: 539-543.

Tomlin, E. S., R. I. Alfaro, J. H. Borden and F. He (1997): Histological response of resistant and susceptible white spruce to simulated white pine damage. Tree Physiol. 18: 21-28.

Tomlin, E. S., E. Antonejevic, R. I. Alfaro and J. H. BORDEN (2000): Changes in volatile terpene and diterpene resin acid composition of resistant and susceptible white spruce leaders exposed to simulated white pine weevil damage. Tree Physiol. 20: 1087-1095. 